\title{
HOLOGRAPHIC EVALUATION OF FIBER OPTICS IMAGE TRANSFER CHARACTERISTICS
}

\section{by}

Paui W. Remijan

A Thesis Submitted to the Faculty of the COMMITTEE ON OPTICAL .SCIENCES

In Partial Fulfillment of the Requirements For the Degree of MASTER OF SCIENCE

In the Graduate Collège THE UNIVERSITY OF ARIZONA 
This thesis has been submitted in partial fulfillment of requircments for an advanced degree at The University of Arizona and is deposited in the University Library to be made available to borrowers under rules of the Library.

Brief quotations from this thesis are allowable without special permission, provided that accurate acknowledgment of source is made. Requests for permission for extended quotation from or reproduction of this manuscript in whole or in part may be granted by the head of the major department or the Dean of the Graduate College when in his judgment the proposed use of the material is in the interests of scholarship. In all other instances, however, permission must be obtained from the author.

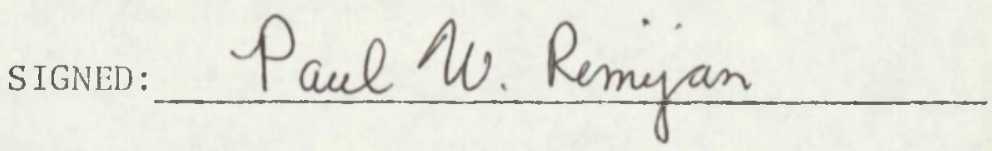

\section{APPROVAL BY THESIS DIRECTOR}

This thesis has been approved on the date shown below:

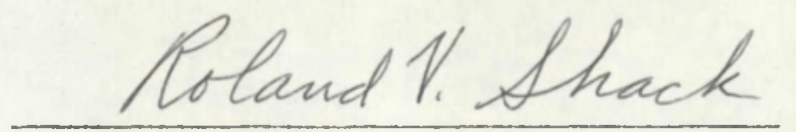

ROLAND V. SHACK

Professor of Optical Sciences
19 JAN 1971

Date 


\section{ACKNOWLEDGMENTS}

The author would like to thank ITEK Corporation for the use of their facilities in performing the experiment. To Dr. James Wyant of. ITEK Corporation goes much appreciation for his suggestions concerning the design of critical components of the holographic system.

I would also like to thank Mosaic Fabrications, Division of Bendix Corporation for donating the fiber optics faceplates used in the experiment and for providing edge response data on these faceplates.

Finally, I would like to express my gratitude to Dr. Roland Shack, my advisor, whose pertinent comments and penetrating critiques were vital to the completion of this thesis. 
TABLE OF CONTENTS

Page

LIST OF ILLUSTRATIONS . . . . . . . . . . . . . . . . . v ABSTRACT •. . . . . . . . . . . . . . . . . . vi vi

I. INTRODUCTION . . . . . . . . . . . . . . . . . . . . 1

Conventional Methods of Fiber Optics Image Evaluation . . . 1

Holographic Method of Fiber Optics Image Evaluation . . . . 3

II. THE HOLOGRAPHIC SYSTEM . . . . . . . . . . . . . . . . . . 5

Production of Holograms . . . . . . . . . . . . . 6

Input Hologram . . . . . . . . . . . . . . . . 8

Output Hologram . . . . . . . . . . . . . . . . . . . 9

Fraunhofer Diffraction Patterns And Their Measurement . . . 11

Fraunhofer Diffraction Patterns From Input Holograms . 12

Fraunhofer Diffraction Patterns From Output Holograms . 13

Measurement of Statistical MTF Values . . . . . . . 16

III. EXPERIMENTAL RESULTS AND DISCUSSION . . . . . . . . . . • . . . 20

Data From The Experiment . . . . . . . . . . . . 20

Errors of The Experiment . . . . . . . . . . . . . 28

Physical Limitations . . . . . . . . . . . . . . 29

IV. COMPARISON OF THE HOLOGRAPHIC METHOD WITH CONVENTIONAL

METHODS OF FIBER OPTICS EVALUATION . . . . . . . . . . . . . . 31

Static Tests . . . . . . . . . . . . . . . . 31

Dynamic Tests . . . . . . . . . . . . . . . 33

Statistical MTF . . . . . . . . . . . . . . . 34

REFERENCES . . . . . . . . . . . . . . . . . . 35 


\section{LIST OF ILLUSTRATIONS}

Figure

Page

1. Fiber Optics Faceplate With 6 Micron Cores . . . . . . . . . . 2

2. System For Producing Holograms . . . . . . . . . . . . . 6

3. Subject A . . . . . . . . . . . . . . . . . . 7

4. Comparison of Input And Output Holograms . . . . . . . . . . 10

5. Fraunhofer Diffraction System . . . . . . . . . . . . . . 11

6. Fraunhofer Diffraction Pattern of An Input Hologram . . . . . 14

7. Transform of Fiber Optics Array . . . . . . . . . . . . 15

8. Fraunhofer Diffraction Pattern of An Output Hologram . . . . . 17

9. "Comparison of "Faceplate Surfaces . . . . . . . . . . . 21

10. Ealing Bar Target . . . . . . . . . . . . . . . . 22

11. Images of 10-100 $\mathrm{Ip} / \mathrm{mm}$ Target With Faceplate P....... 23

12. Images of 100-1000 1p/mm Target With Faceplate P. . . . . . 24

13. Images of Target With Faceplate G . . . . . . . . . . . . . 25

14. Statistical MTF . . . . . . . . . . . . . 26

15. Fraunhofer Diffraction Pattern of Faceplate $P$ Output Hologram . . . . . . . . . . . . . 27

16. Edge Response Transfer Function versus Statistical MTF . . . 32 


\begin{abstract}
A fiber optics image-transferring element is non-isoplanatic over a surface region containing only a few fundamental resolution elements and no consistent or meaningful evaluation of a fiber optics image can be made by examining such a small non-isoplanatic region. It is proposed that a fiber optics image be considered as an ergodic statisti= cal ensemble where each member of the statistical ensemble is one output from one faceplate. Ergodicity of the ensemble image allows the necessary statistical information about the image to be obtained from the measurement of the average properties of one output. With image formation considered as a stochastic process, the fiber optics element can be described by a statistical transfer function.

A holographic system is used to create an input sinusoidal irradiance distribution. Subsequent holographic evaluation of the amplitude of the sinusoidal input object and the average amplitude of the sinusoidal output component results in the direct calculation of a statistical MTF. The design and operation of such a holographic system is presented and an experiment which demonstrates the advantages of the holographic measurement is discussed.
\end{abstract}


CHAPTER I

INTRODUCTION

An image-transferring fiber optics element consists of an ordered array of small individual "light pipes". The ordexed axray of "light pipes" maintains the spatial relationship between light and dark regions of an object at the input surface and thus produces an image at the output surface. Image-transferring fiber optics elements and evaluation of the images they produce are the topic of this paper.

The evaluation of the image-forming process for a linear, isoplanatic optical element is quite adequately expressed by an optical transfer function, and with some difficulty, optical transfer functions and modulation transfer functions for lenses can be obtained experimentally. Fiber optics image evaluation requires special consideration, because a fiber optics, element is non-isoplanatic over a surface region of only a few single fibers. The existence of discontinuous and inconsistent spread functions over the grossly non-isoplanatic fiber optics surface means that a meaningful, consistent transfer function cannot be calculated by usual methods.

\section{Conventional Methods of Fiber Optics Image Evaluation.}

Conventional methods of investigating the fiber optics image transfer process utilize the spread function and edge response as a basis for a transfer function calculation. An examination of Fig. 1 


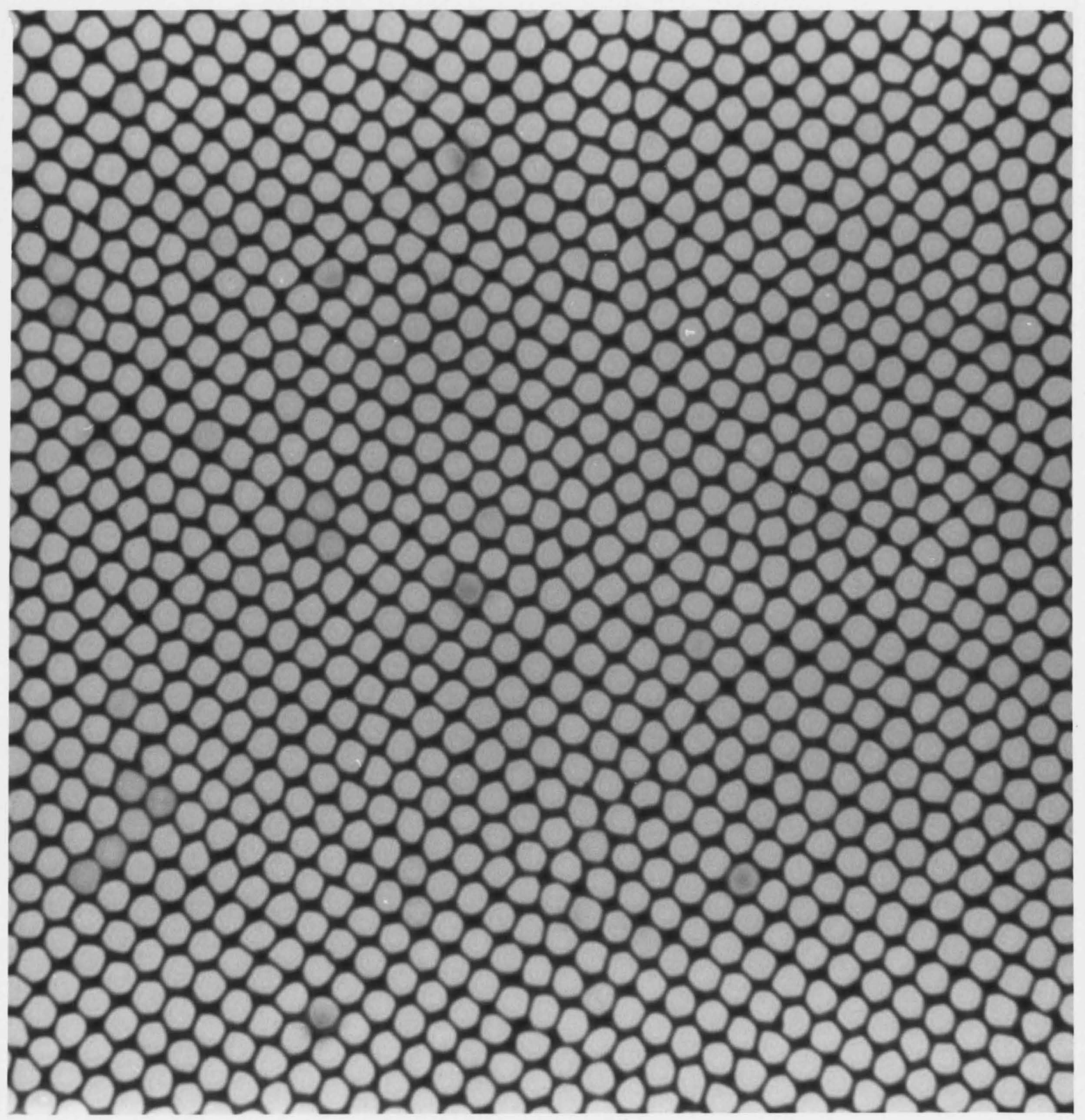

Fig. 1 Fiber Optics Faceplate With 6 Micron Cores 
reveals how an edge, line or point response will depend on the orientation and location of an edge, line or point object with respect to fiber striations and surface discontinuities. A transfer function calculated for such an obviously non-isoplanatic region does not have a clear meaning.

Holographic Method of Fiber Optics Image Evaluation

Holographic testing of fiber optics faceplates and the calculation of statistical MTF requires a statistical consideration of the fiber optics image formation process. Consider the "image" formed by a. faceplate to be a collection of outputs where each output is associated with a particular faceplate of an ensemble. Each element of the faceplate ensemble is assumed to be consistent in physical size, material composition, fiber size and location-orientation with respect to the input. The elements of the faceplate ensemble are assumed to be random in properties such as array distortions, blemishes, dead single fibers and cross-talk. Next, it is assumed that, the stochastic image-forming process is ergodic and it is necessary to measure the average properties of only one output in order to determine the average properties of the more general "image".

The. first step of the holographic evaluation process applies a continuous, spatially periodic, sinusoidal irradiance distribution to the input surface of a fiber optics element. It is desirable to apply the imput to a large fraction of the surface area of the faceplate so that the average properties of an experimental output will closely approximate the average properties of one output of the "image" ensemble. 
- The second step of the holographic process measures the average amplitude of the sinusoidal component in one output. The amplitude of the sinusoidal input is a1so measured in an identical manner. A comparison of the average output amplitude to the input amplitude yields a transfer factor. Using well known techniques it is possible to apply a summation of sinusoidal irradiance distributions of various spatial frequencies as an input and to measure a transfer factor for each sinusoidal irradiance distribution. Each transfer factor is a value of a meaningfuI, consistent, statistical MTF. 


\section{CHAPTER II}

\section{THE HOLOGRAPHIC SYSTEM}

The basic holographic system used to evaluate fiber optics image transfer is quite simple. First, a summation of sinusoidal irradiance variations are applied to the entire input surface of the fiber optics. A photographic plate is used to record the summation of sinusoidal fringes at the input surface and the output surface of the fiber optics, thus producing an input hologram and output hologram. Next, the Fraunhofer diffraction patterns of each of the two holograms are produced. The irradiances of the narrow sinc ${ }^{2}$ light distributions in the respective Fraunhofer diffraction pattems are directly related to the amplitude of the sinusoidal irradiance variations that are recorded on each hologram. Since the amplitudes of the sinusoidal patterns on the output hologram are those on the input hologram modified by the fiber optics element, a comparison of $\operatorname{sinc}^{2}$ irradiances in the respective Fraunhofer patterns yields a fiber optics transfer factor for each of the input sinusoidal intensity variations.

The proper operation of such a holographic system is dependent upon very closely approximating a pure amplitude hologram which is linear in amplitude transmission versus exposure. 
Production of Holograms

Fig. 2 shows the basic system for producing input and output holograms.

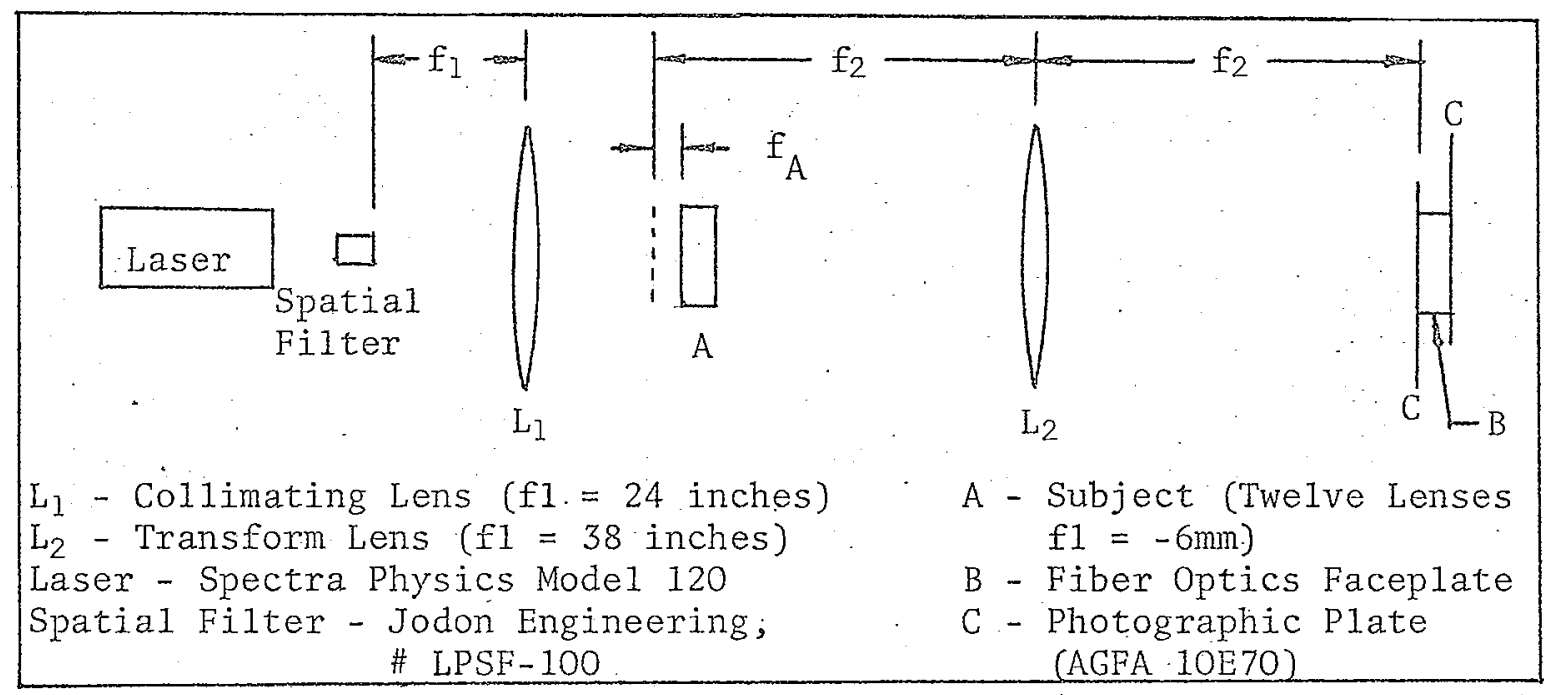

Fig. 2 System For Producing Holograms

The output hologram is made by pressing a photographic plate against the output surface of the fiber optics faceplate. The faceplate is then removed and the input hologram is made by placing the photographic plate at the position of the fiber optics input surface. To keep the development process problems under control, both the input and output holograms are recorded on the same plate.

The subject in the above diagram is the heart of the system for producing holograms. Twelve commercially available double concave lenses of $8 \mathrm{~mm}$ diameter and $-6 \mathrm{~mm}$ focal length were ground into rectangular shapes of varying widths. The rectangular lenses were mounted in a specially machined holder. A photograph of the holder with lenses appears in Fig. 3. Eleven of the lenses are covered with a neutral density 


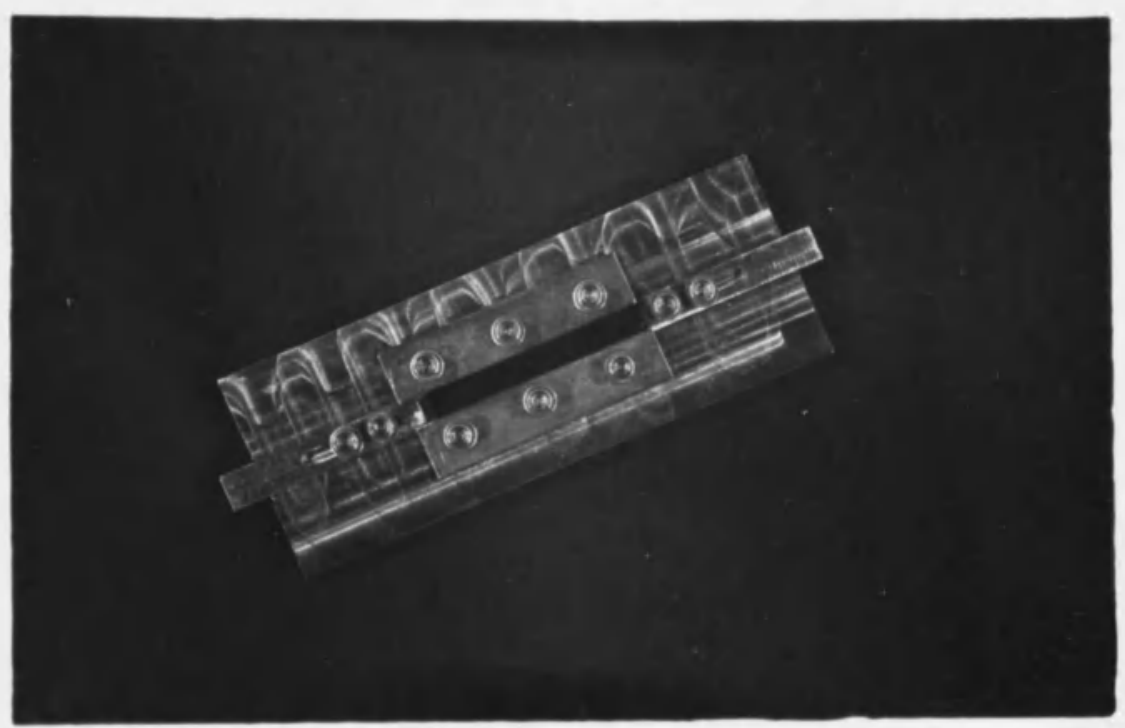

a. Side Facing $\mathrm{L}_{1}$

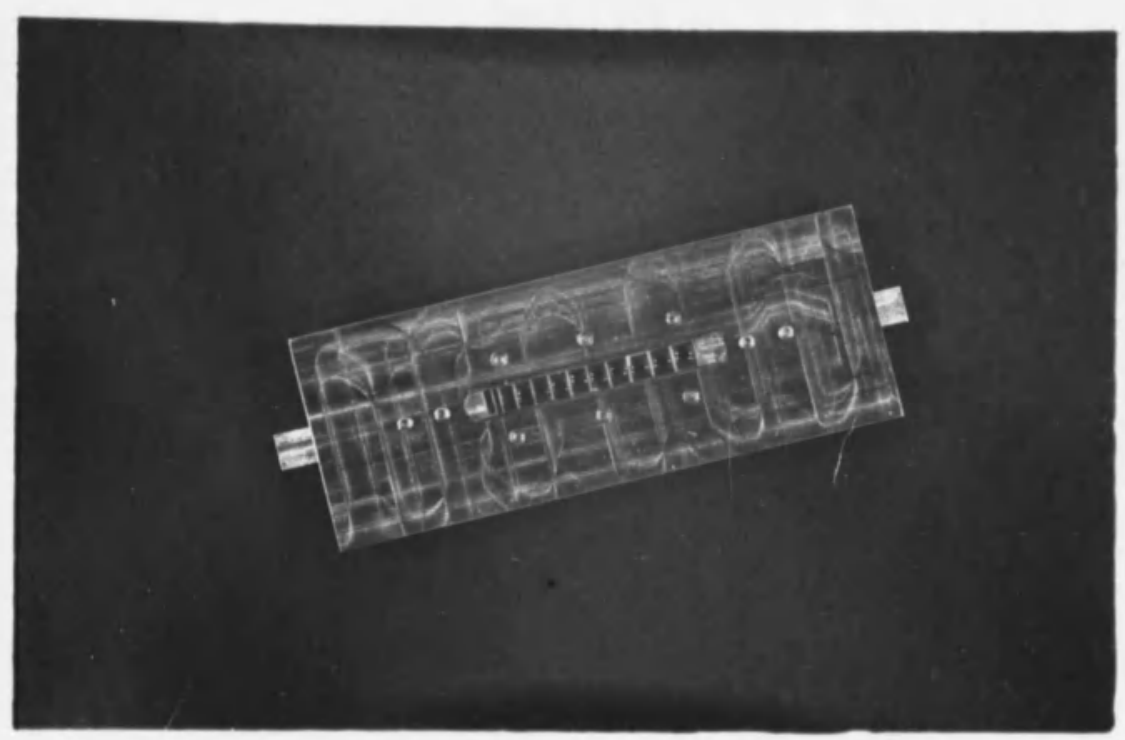

b. Side Facing $\mathrm{L}_{2}$

Fig. 3 Subject A 
filter, N. D. $=3.0$, on the side of the subject exposed to collimated light from $L_{l}$. One lens is left uncovered to produce a reference wave. Collimated monochromatic light passes through the negative lenses and appears to diverge from the focal points of the negative lenses. The next lens $\mathrm{L}_{2}$ is located so that its front focal plane contains the vixtual point sources formed $6 \mathrm{~mm}$ to the left of the twelve negative lenses. On the other side of $L_{2}$, plane waves from each of the eleven virtual object points interfere with the reference plane wave producing an irradiance distribution containing eleven distinct sinusoidal components. The spatial frequencies of these sinusoidal components are readily tabulated from geometxical considerations. The spatial frequency of the pattern associated with each lens is

$$
\varepsilon_{i}=\frac{S_{i}}{f_{2} \lambda} \text { cycles/unit length, }
$$

with $S_{i}$ denoting the center-to-center spacing between the reference lens and any of the other eleven negative lenses. The $f$ /number of the twelve negative lenses is kept small enough to allow a common region of interference for all twelve lenses.

Input Hologram

A photographic plate placed in the input plane of the fiber optics records the summation of sinusoidal intensity variations created by the subject $A$ and transform lens $\mathrm{L}_{2}$. Upon normal exposure and development, a photographic plate is non-linear in amplitude transmission versus exposure, but at low exposure and low modulation the desired Iinearity of amplitude transmission versus exposure is obtained (Kozma 1966; 
Goodman 1968, pp. 230-241). The use of a very thin 7 micron emulsion also helps to reduce the possibility of having excess index variations and surface irregularities which cause a phase hologram.

\section{Output Hologram}

A photographic plate placed in the output plane of the fiber optics records the input irradiance distribution after modification by the fiber optics. Unique properties of the fiber optics allow satisfactory production of the output hologxam (Potter 1961). Basically, light incident on fibers at a small angle emerges at the same small angle. This fact coupled with the use of a very thin 7 micron emulsion allows the photographic plate to record the energy transmitted by each fiber separately, with no overlapping. Exposure time for the output hologram is approximately $10 \%$ to $20 \%$ Ionger than for the input hologram because of transmission losses in the fiber optics faceplate. Fig. 4 is a series of three photographs showing an input hologram, output holo- gram, and fiber optics faceplate, all at the same magnification. A comparison of the second and third photographs shows the independent energy transfer for each single fiber that causes the output hologram to be a recording of "intensity spots".

Here it is appropriate to point out the significance of the "intensity spots" recorded at the fiber. optics output surface. Upon closer examination each output "intensity spot" has a very high frequency speckle appearance due to coherence within a single fiber, but this has no effect upon resolving the lower spatial frequency input used in the experiment. With low angles of illumination and the use of a very thin 


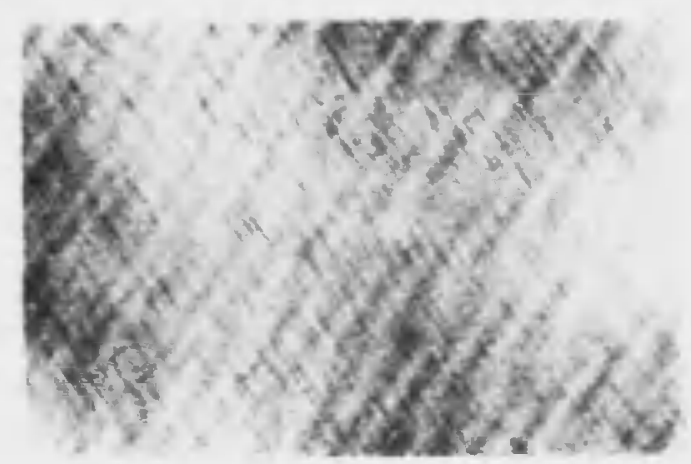

a. Input Hologram

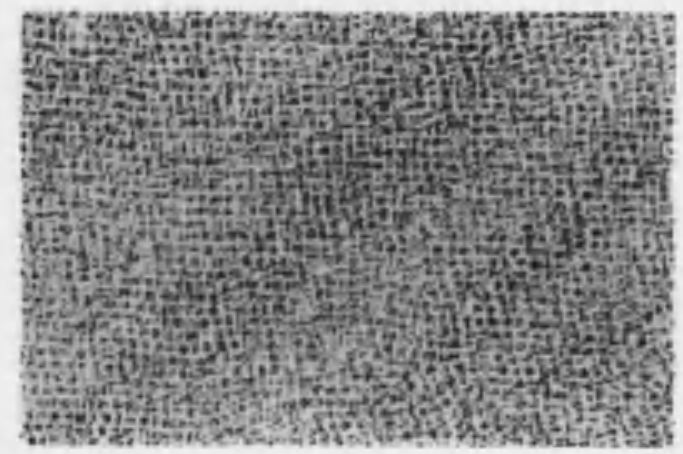

b. Output Hologram

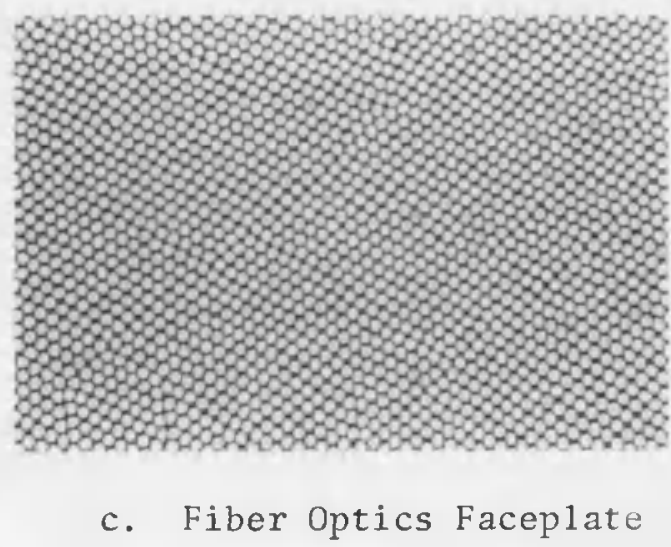

Fig. 4 Comparison of Input And Output Holograms 
emulsion, a complete spatial separation of individual "iritensity spots" is achieved. For a given input, an output hologram would show a spatially identical collection of non-overlapping "intensity spots" for both coherent and incoherent illumination. This means that the evaluation of the fiber optics imaging process and the calculation of a statistical transfer function is essentially the evaluation of an incoherent statistical transfer function.

Fraunhofer Diffraction Patterns And Their Measurement

The Fraunhofer diffraction patterns produced by the output hologram and the input hologram are examined in the second part of the holographic scheme.

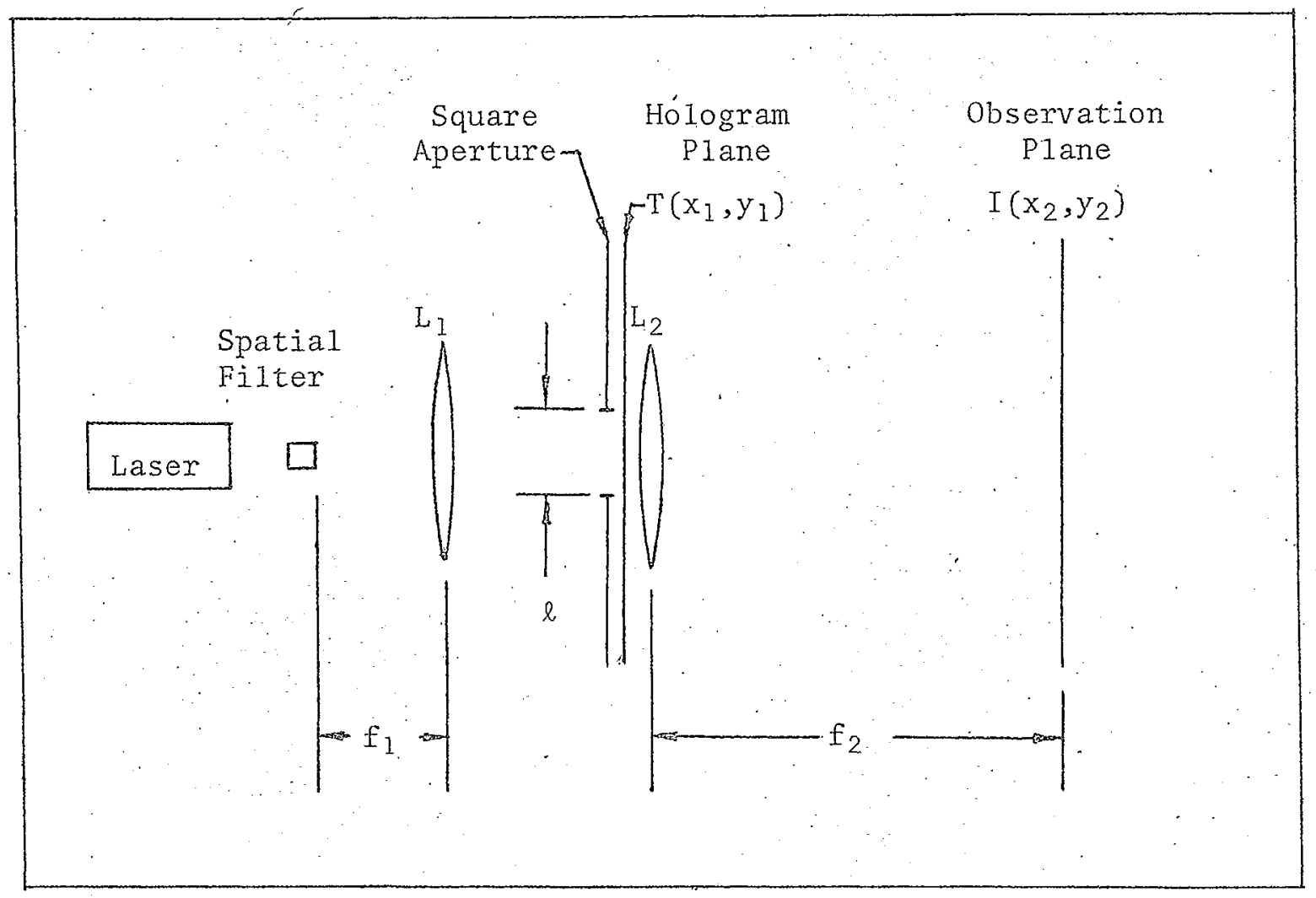

Fig. 5 Fraunhofer Diffraction System 
Apart from a quadratic phase factor the complex amplitude distribution in the $x_{2}, y_{2}$ plane is just the Fourier transform of the transmission function $T\left(x_{1}, y_{1}\right)$. The calculations of the Fraunhofer diffraction patterns are also independent of the quadratic phase factor of the complex amplitude distribution, and again are just straightforward Fourier transform calculations. Other unimportant constants are not shown explicitly in the final expressions for the Fraunhofer diffraction patterns.

Fraunhofer Diffraction Patterns From Input Holograms

The transmission function for the input hologram is written as

$$
\mathrm{T}_{I}\left(\mathrm{x}_{1}, \mathrm{y}_{I}\right)=\left[\mathrm{K}+\sum_{i=1}^{1 l} \mathrm{~m}_{i I} \cos \left(2 \pi \varepsilon_{i} \mathrm{x}_{I}\right)\right]\left[\operatorname{rect}\left(\frac{\mathrm{x}_{I}}{\ell}\right) \operatorname{rect}\left(\frac{y_{l}}{\ell}\right)\right],
$$

e is the width of a square pupil which limits the input irradiance distribution and $\mathrm{K}$ is a background which is approximately 1000 times $\mathrm{m}_{i}$, the amplitude of a sinusoidal transmission variation. Subscript I refers to the input hologram. The Fraunhofer diffraction pattern for $\mathrm{T}_{I}\left(\mathrm{x}_{1}, \mathrm{y}_{1}\right)$ is given by

$$
\begin{aligned}
& I_{I}\left(x_{2}, y_{2}\right)=K^{2} \ell^{4} \operatorname{sinc}^{2}\left(\frac{l x_{2}}{\lambda f_{2}}\right) \operatorname{sinc}^{2}\left(\frac{l y_{2}}{\lambda f_{2}}\right)+. \\
& \sum_{i=1}^{11} \frac{\ell^{4} m_{i I}}{4}\left[\operatorname{sinc}^{2}\left\{\frac{\ell}{\lambda f_{2}}\left(x_{2} \pm \varepsilon_{i} \lambda f_{2}\right)\right\}\right]\left[\operatorname{sinc}^{2}\left(\frac{l y_{2}}{\lambda f_{2}}\right)\right] .
\end{aligned}
$$

There is a very narrow two-dimensional sinc ${ }^{2}$ function at the origin plus very narrow two-dimensional sinc ${ }^{2}$ functions centered at 
$\pm \varepsilon_{i} \lambda f_{2}$ : Due to the low modulation levels used to make the hologram, the magnitude $l^{4} \mathrm{~K}^{2}$ of the central sinc ${ }^{2}$ function is at least four thousand times $i^{4} m_{i}{ }^{2 / 4}$. Fig. 6 is a photograph of a diffraction pattern from an input hologram. The important observation in Fig. 6 is the absence of any measurable higher order harmonics, and the absence of any patterns which would be caused by a phase modulation term in $T_{I}\left(x_{1}, y_{1}\right)$, '(Goodman 1968; pp. 69-70).

Fraunhofer Diffraction Patterns From Output Holograms

As shown in Fig. 1, the fiber optics array is basically a hexagonal array of single fibers with the diameters of the single fibers very nearly equal to their center-to-center spacing. It is the hexagonal array of circular light pipes of radius a, which modulates the sinusojdal patterns at the input surface. The amplitude transmission for the output hologram is indicated with a subscript 0 , and given by

$$
\begin{gathered}
\mathrm{T}_{0}\left(\mathrm{x}_{1}, \mathrm{y}_{1}\right)=\left\{[\text { HEX ARRAY }] *\left[\operatorname{circle} \frac{\mathrm{r}_{1}}{\mathrm{a}}\right]\right\} \\
\left.\left.\qquad \mathrm{K}+\sum_{i=1}^{11} \mathrm{~m}_{i 0} \cos \left(2 \pi \varepsilon_{i} \mathrm{x}_{1}\right)\right]\left[\operatorname{rect}\left(\frac{\mathrm{x}_{1}}{\ell}\right) \operatorname{rect}\left(\frac{\mathrm{y}_{1}}{\ell}\right)\right]\right\} .
\end{gathered}
$$

The transform of the fiber optics array is a hexagonal array of delta functions multiplied by the circle function transform. Except for the convolution term from the fiber optics array transform, the Fraunhofer diffraction pattern from the output hologram has the same form as $I_{I}\left(x_{2}, y_{2}\right)$, and is written as 


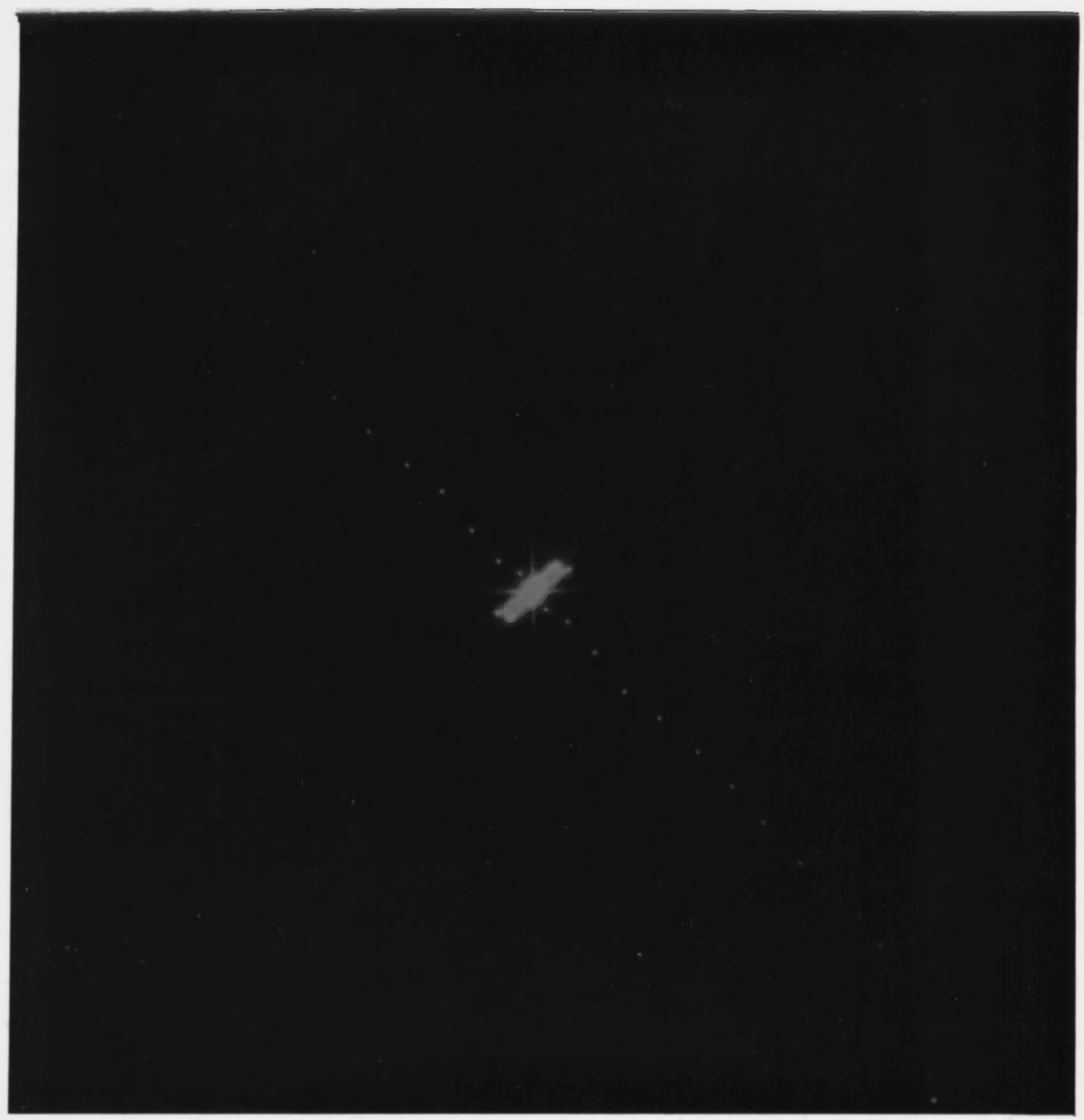

Fig. 6 Fraunhofer Diffraction Pattern of An Input Hologram 


$$
\begin{aligned}
& I_{0}\left(x_{2}, y_{2}\right)=\left\{[\text { HEX ARRAY }]\left[\frac{a^{2} J_{1}\left(\frac{2 \pi a r_{2}}{\lambda f_{2}}\right)}{\operatorname{ar}_{2}}\right\} *\right. \\
& \lambda \mathrm{f}_{2} \\
& \left\{\mathrm{~K}^{2} \ell^{4} \operatorname{sinc}^{2}\left(\frac{l \mathrm{x}_{2}}{\lambda \mathrm{f}_{2}}\right) \operatorname{sinc}^{2}\left(\frac{l y_{2}}{\lambda f_{2}}\right)+\right. \\
& \left.\sum_{i=1}^{11} \frac{\ell^{4} \mathrm{~m}_{i 0^{2}}}{4}\left[\operatorname{sinc}^{2} \frac{\ell}{\lambda f_{2}}\left(x_{2} \pm \varepsilon_{i} \lambda f_{2}\right)\right]\left[\operatorname{sinc}^{2}\left(\frac{l y_{2}}{\lambda f_{2}}\right)\right]\right\}
\end{aligned}
$$

Fig. 7 shows the first term of $I_{0}\left(x_{2}, y_{2}\right)$. More hexagonally distributed

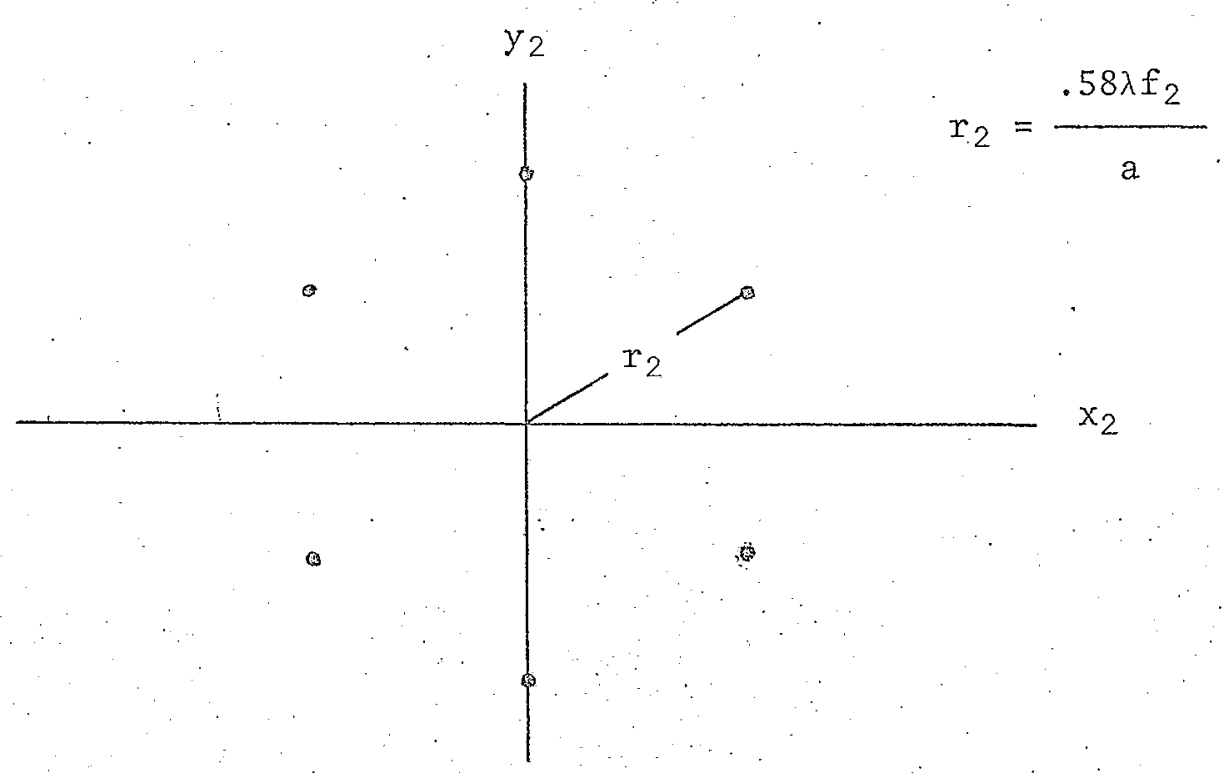

Fig. 7. Transform of Fiber Optics Array

delta functions are generated by the transform of the hexagonal array, but multiplication by the circle transform rendexs the products 
insignificant. Even the six outer dots shown in Fig. 7 are very weak in comparison to the central irradiance because of the circle transform cutoff at

$$
r_{2}=\frac{.61 \lambda f_{2}}{a}
$$

The first term of $I_{0}\left(x_{2}, y_{2}\right)$ has a dominant term at the origin of the hexagonal array, while the second term of $I_{0}\left(x_{2}, y_{2}\right)$ has a dominant central intensity with $\mathrm{K}^{2} \ell^{4}$ much greater that $\ell^{4+m_{0}} 2 / 4$. With two dominant terms at the origin, the convolution, $I_{0}\left(x_{2}, y_{2}\right)$, reduces to a superposition of the function shown in Fig. 7 with a function of the form of Fig. 6. Fig. 8 is a photograph of a Fraunhofer diffraction pattern from an output hologram. As shown by the photograph, those portions of the pattern associated with the hexagonal array are barely visible and do not interfexe with the narrow sinc ${ }^{2}$ functions which are associated with the sinusoidal amplitude transmission variations of the output hologram.

Further examination of Fig. 8 reveals the absence of sinc $^{2}$ irradiances ten and eleven. The input sinusoidal irradiance distributions required to produce higher frequency sinusoidal amplitude transmissions in the output hologram are not transmitted by the faceplate.

Measurement of Statistical MTF Values.

The most important features of the Fraunhofer diffraction patterns produced by input and output holograms are the narrow sinc ${ }^{2}$ irradiances caused by the sinusoidal amplitude transmission variations of 


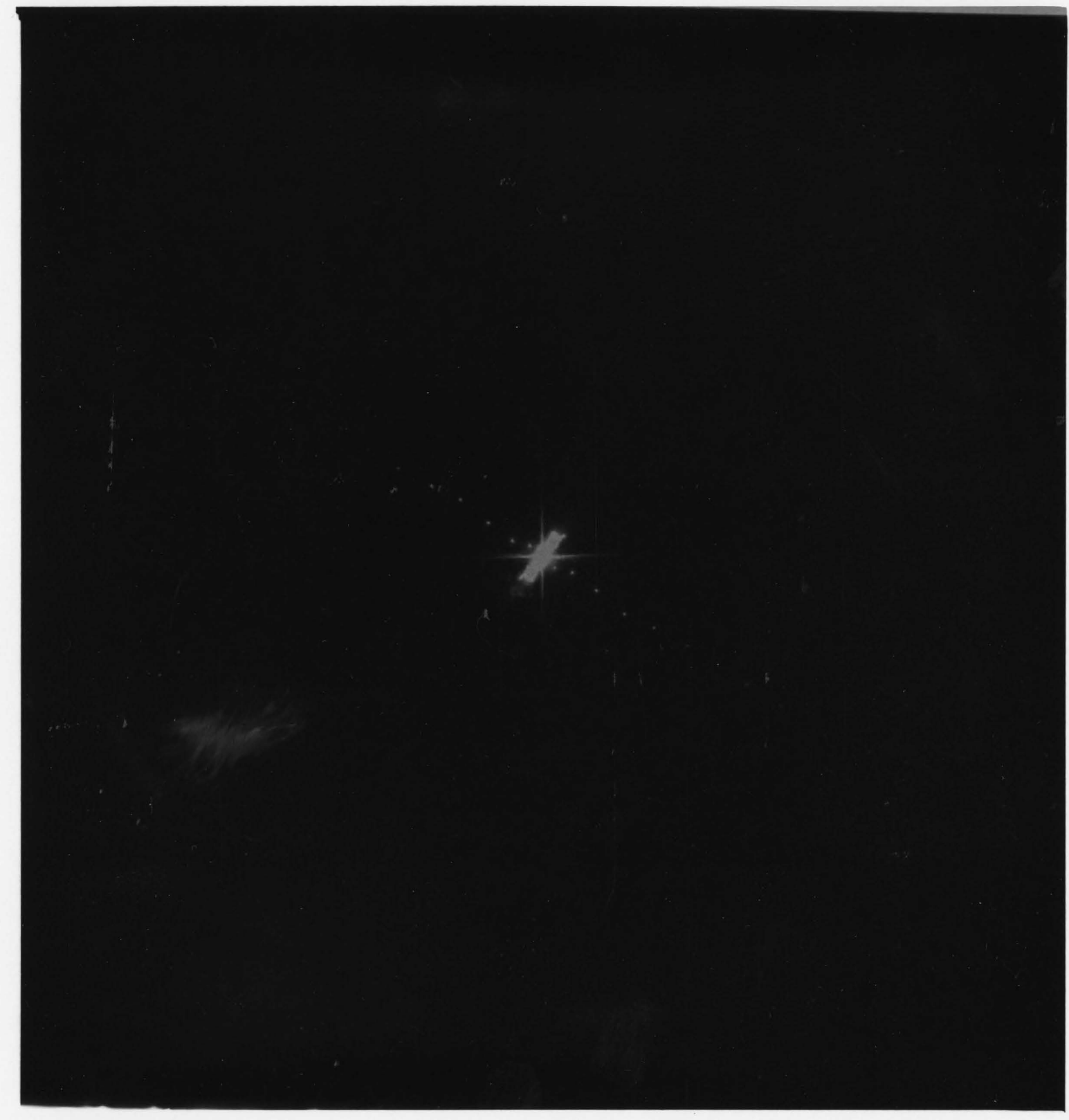

Fig. 8 Fraunhofer Diffraction Pattern of An Output Hologram 
the input and output holograms. Looking at the expressions for $I_{I}\left(x_{2}, y_{2}\right)$ and $I_{0}\left(x_{2}, y_{2}\right)$, Eqs. (3) and (5), the narrow sinc ${ }^{2}$ irradiances of the Fraunhofer diffraction patterns are proportional to $\mathrm{m}_{i \mathrm{I}}{ }^{2}$ and $\mathrm{m}_{i 0}{ }^{2}$, the squared magnitude of the sinusoidal amplitude transmission variations. The sinc ${ }^{2}$ irradiances in the diffraction pattern from the input hologram are measured with a PMT and the sinc2 irradiance corresponding to the lowest spatial frequency amplitude transmission variation of the input hologram is normalized with $\ell^{4} \mathrm{~m}_{1 \mathrm{I}} 2 / 4=1$.' Similarly, the measured sinc2 irradiances in the diffraction pattem caused by the sinusoidal amplitude transmission variations of the output hologram are normalized with $\ell^{4} m_{0} 2 / 4=1$. The experimental statistical MTF values of the fiber optics faceplate are now just

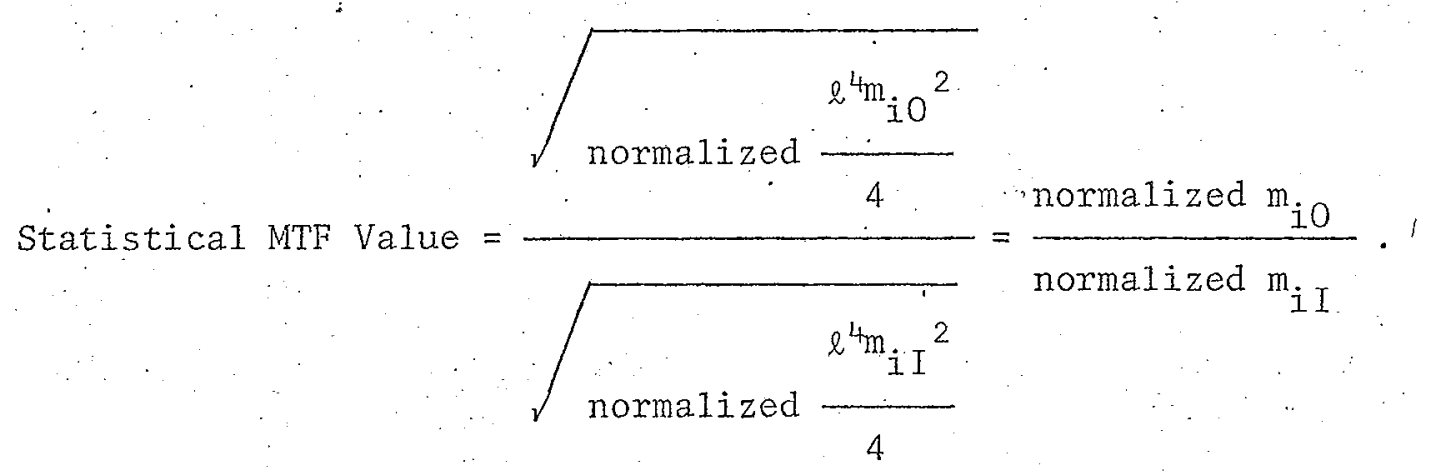

The relative irradiance measurements were made by scanning a Fraunhofer diffraction pattern with a PMT and slit on a transverse carriage. The s.lit width was approximately equal to the width of the main lobe of the sinc ${ }^{2}$ functions. A slit length of approximately ten times the width was chosen to allow for a non-critical vertical positioning of the PMT slit assembly. The exact slit height was relatively 
unimportant since the entire slit exposed to an area in between successive points revealed a signa1-to-noise ratio of greater than one hundred to one: 
CHAPTER III

\section{EXPERTMENTAL RESULTS AND DISCUSSION}

Generally speaking, the experiment produced repeatable statistical MTF values.

\section{Data From The Experiment}

Two faceplates were tested with the holographic system. One sample, faceplate $G$, was à good quality parallelepiped faceplate $3 \mathrm{~cm} \times 2 \mathrm{~cm} \times 0.5 \mathrm{~cm}$. The other sample, faceplate $\mathrm{P}$, was a poor quality cylindrical faceplate with $1 \mathrm{~cm}$ radius and a length of $3 \mathrm{~cm}$. Both faceplates were constructed of the same type of glass and had N.A. = 1.00 , $6 \mu$ cores and approximately the same center-to-center spacing between single fibers.

Fig. 9. compares the surfaces of faceplate $G$ and faceplate. $P$. Further examination of the two faceplates is accomplished with a high contrast Ealing bar target which has three major groups of $1-101 \mathrm{p} / \mathrm{mm}$, 10-100 $\mathrm{lp} / \mathrm{mm}$ and 100-1000 $\mathrm{lp} / \mathrm{mm}$. Fig. 10. shows the high contrast Ealing bar target, while Fig. 11 and Fig. 12 show how the performance of faceplate $P$ depends upon its position with respect to the target. Fig. 13 shows that the good faceplate provides consistent performance except for striation alignment with respect to the resolution bars. It does not suffer from large distortions between input and output surfaces. Statistical MTF values calculated from data obtained with the 


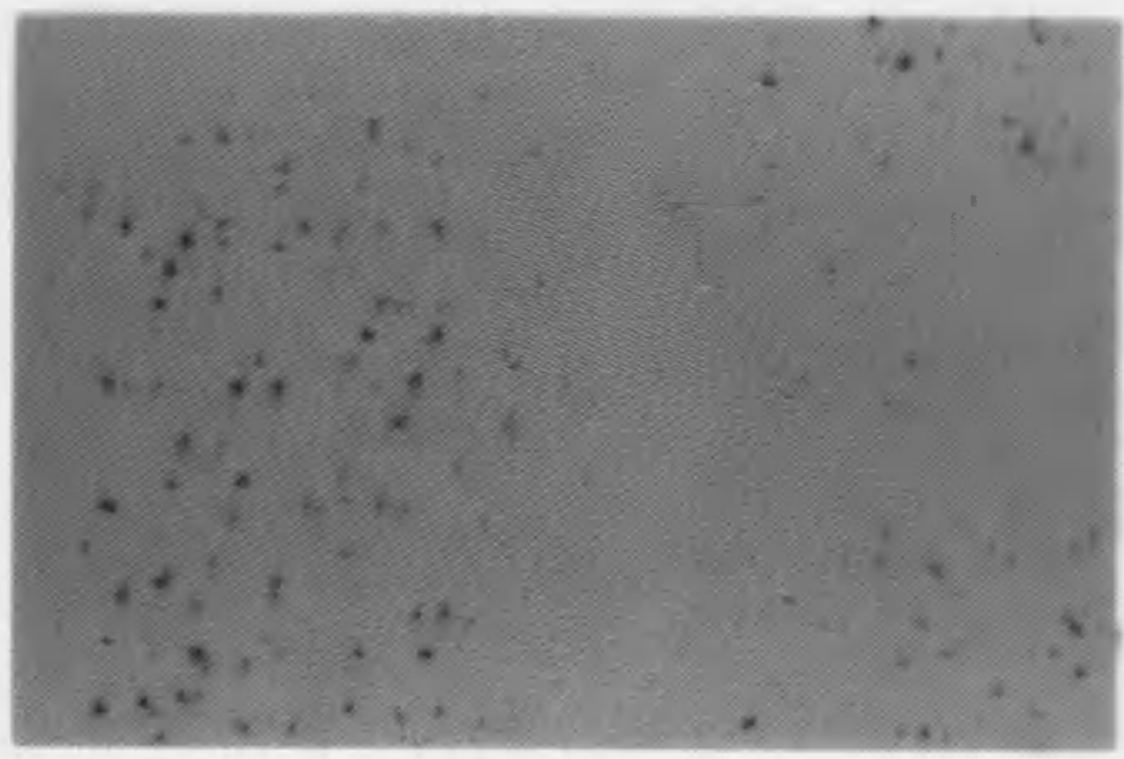

a. Faceplate G

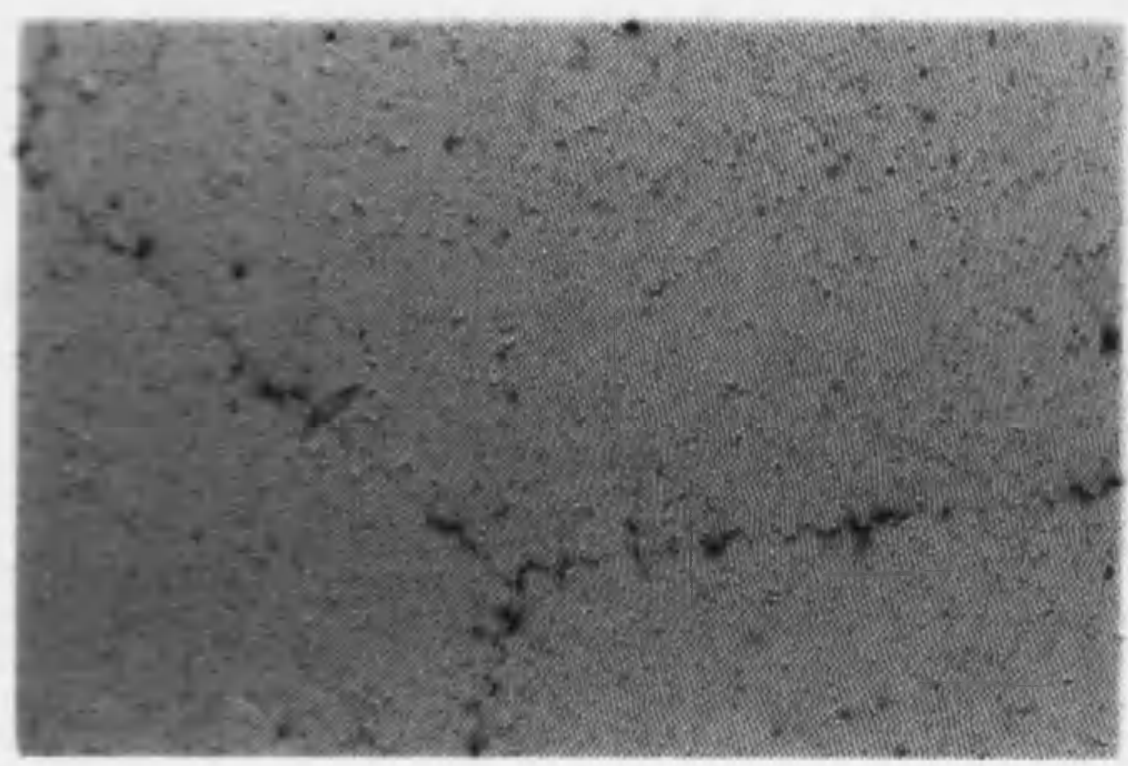

b. Faceplate $P$

Fig. 9 Comparison of Faceplate Surfaces 


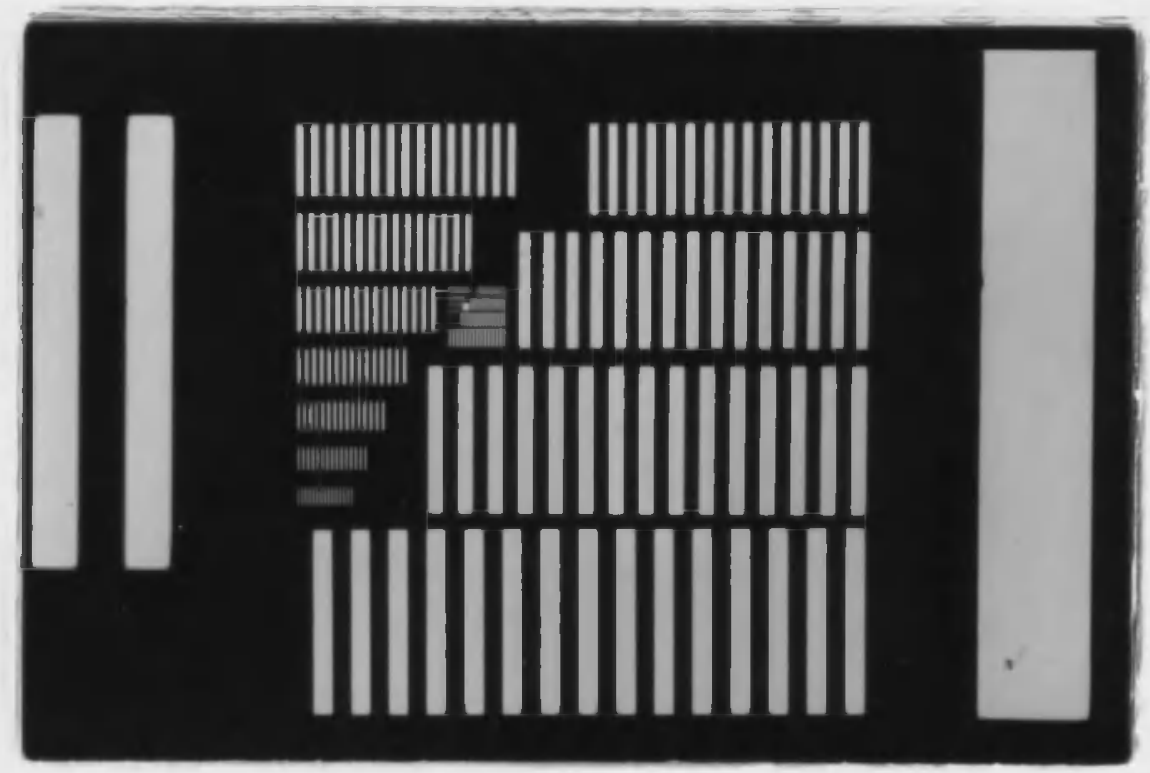

a. $10-1001 \mathrm{p} / \mathrm{mm}$

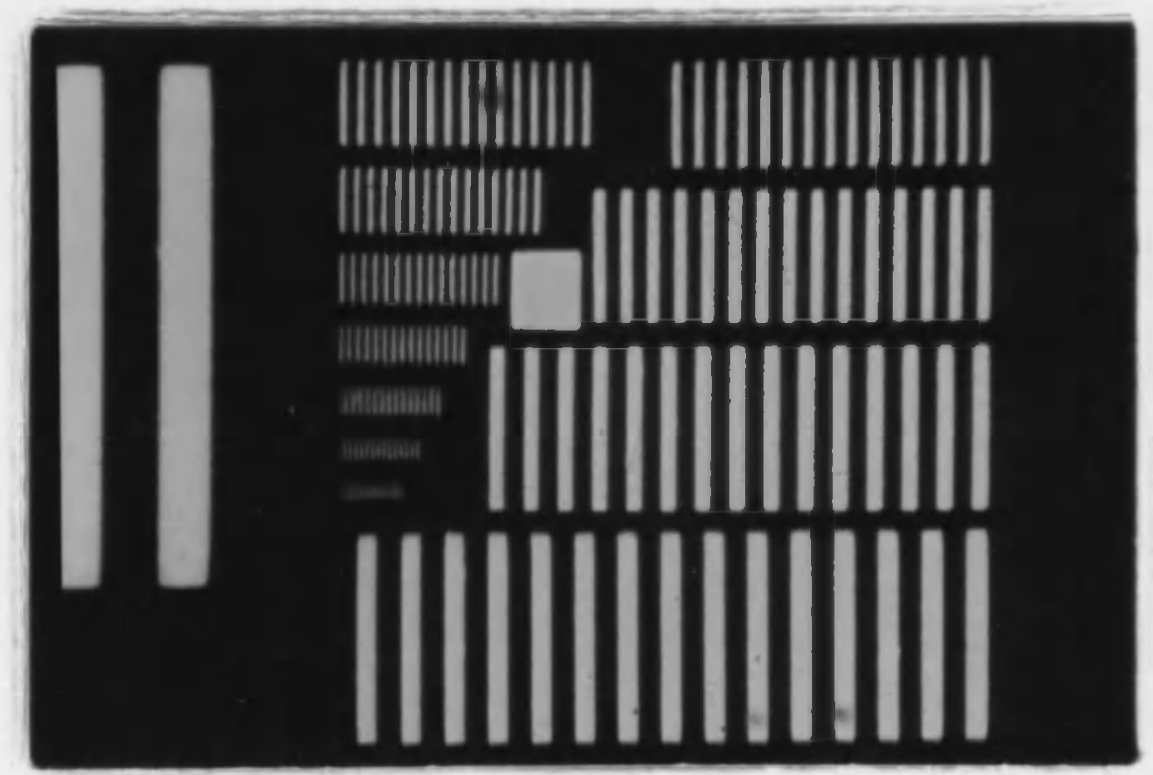

b. $100-1000 \mathrm{lp} / \mathrm{mm}$ 


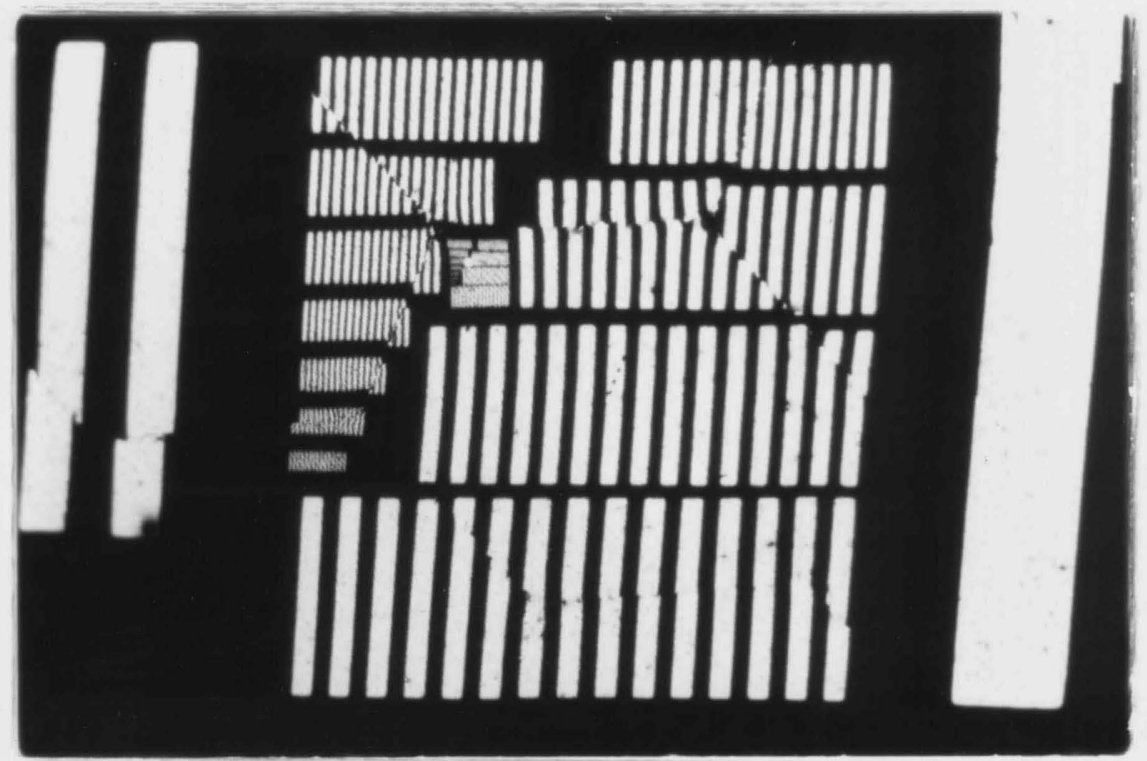

a. Poorest Image

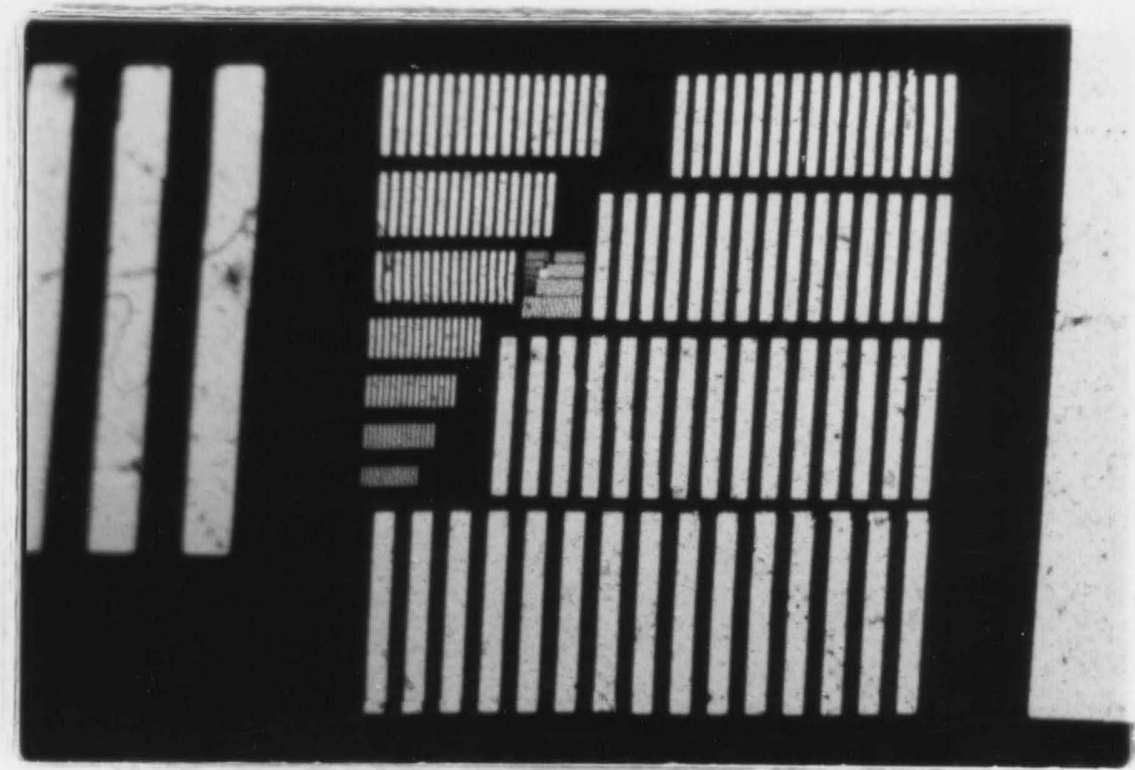

b. Best Image

Fig. 11 Images of $10-1001 \mathrm{p} / \mathrm{mm}$ Target With Faceplate P 


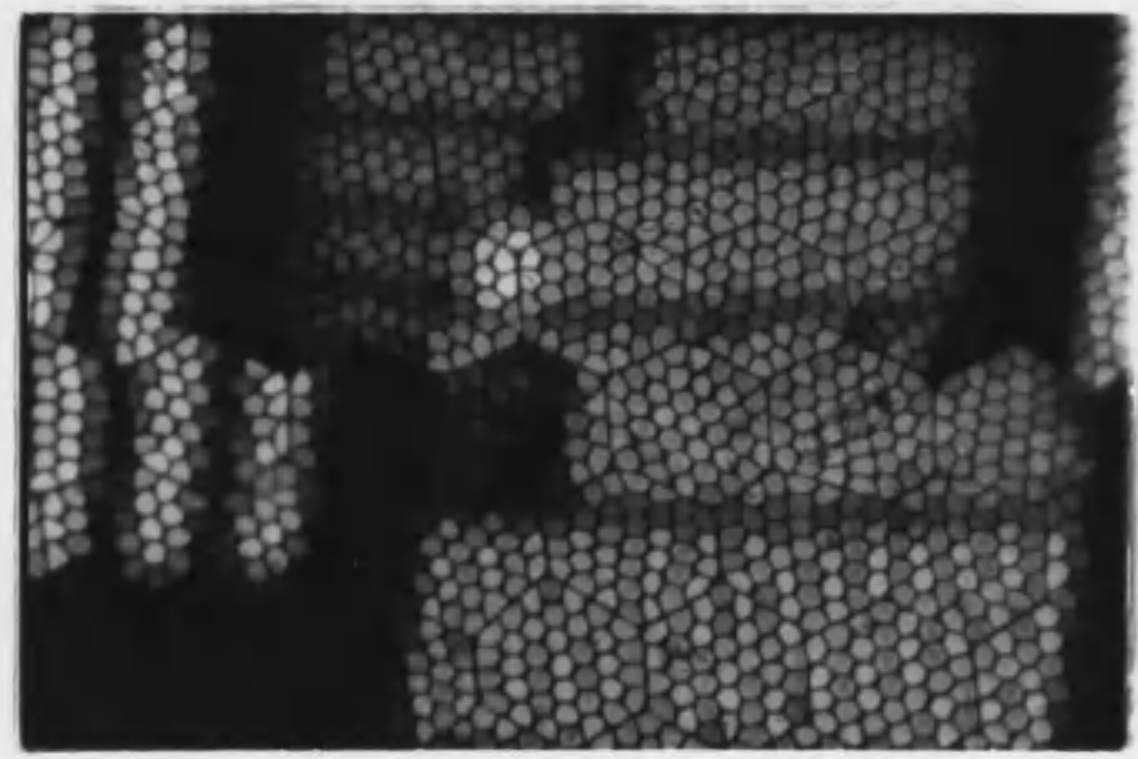

a. Poorest Image

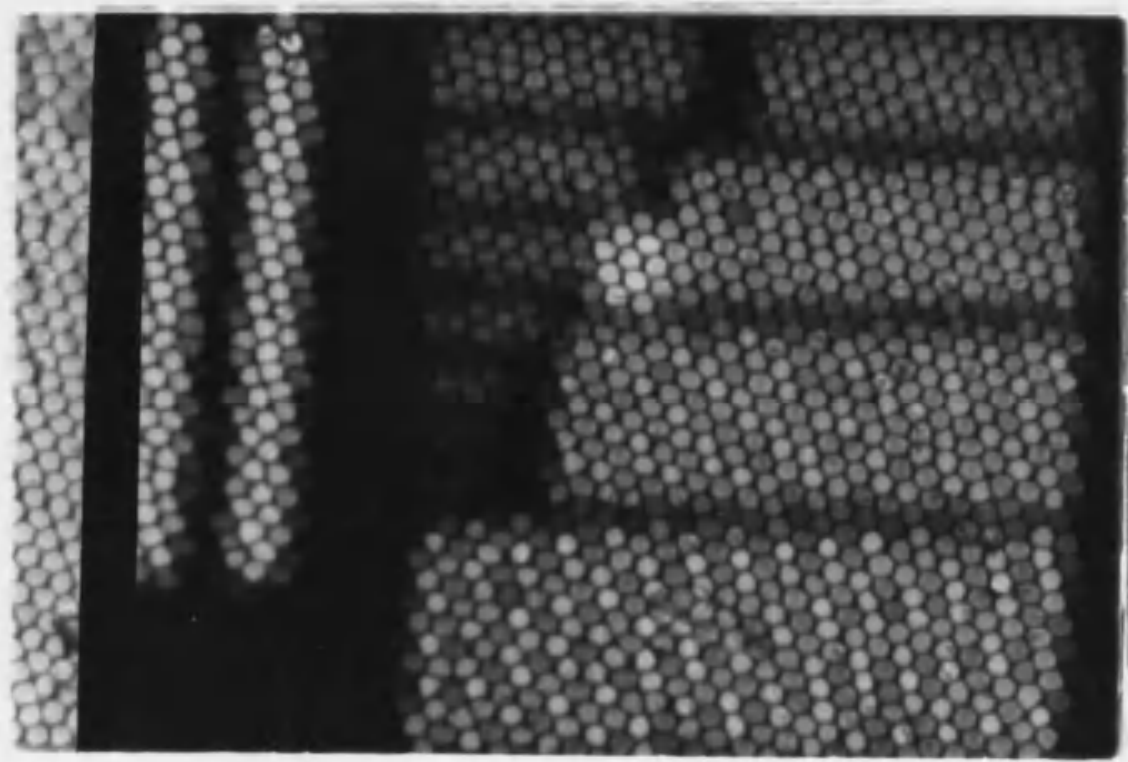

b. Best Image

Fig. 12 Images of $100-1000 \mathrm{lp} / \mathrm{mm}$ Target With Faceplate P 


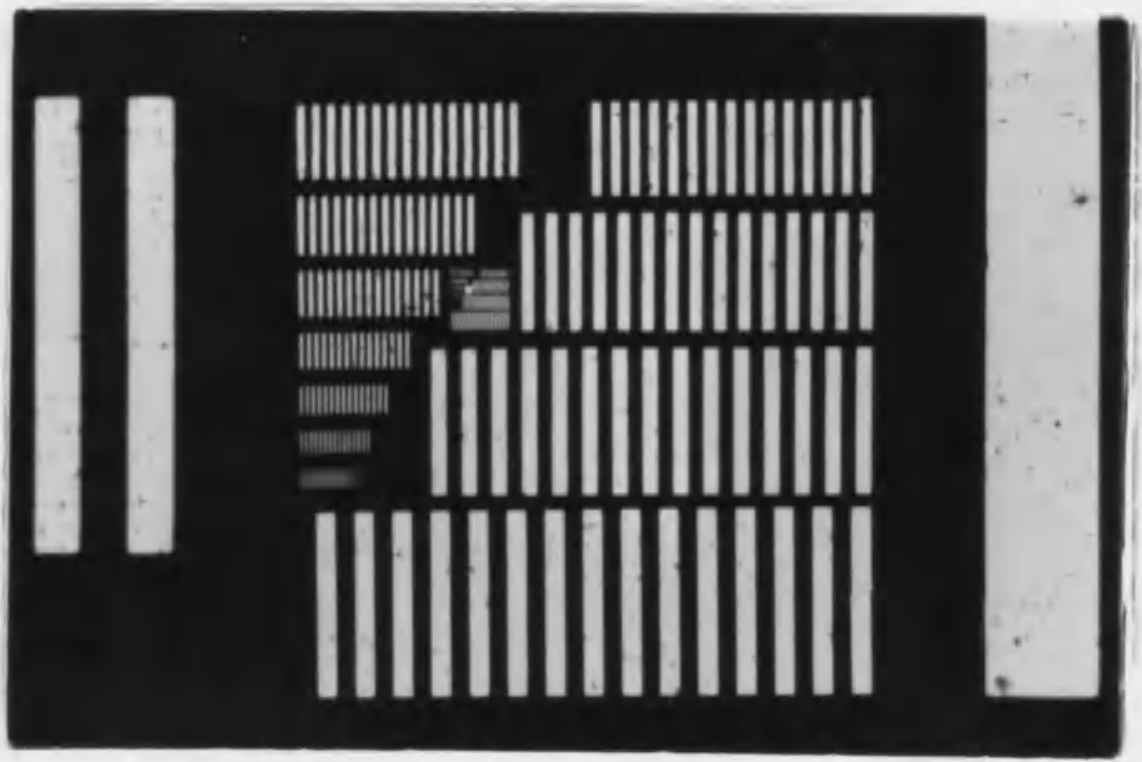

a. Image of $10-1001 \mathrm{p} / \mathrm{mm}$ Target

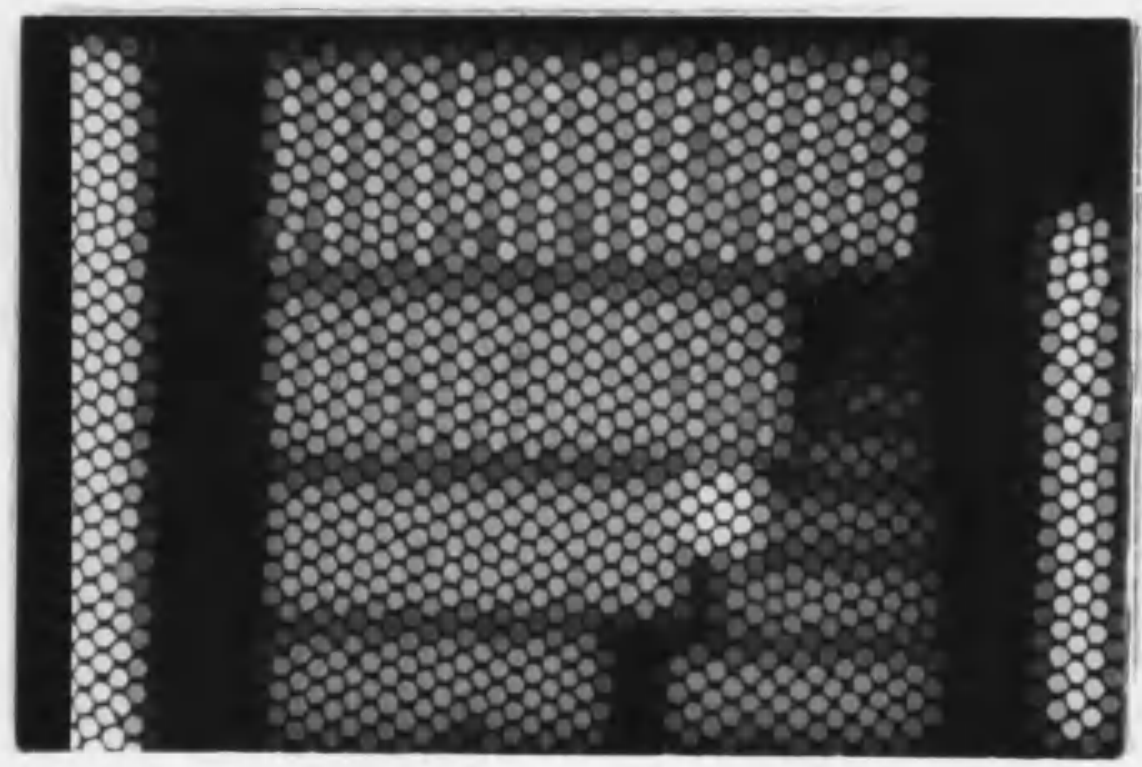

b. Image of 100-1000 1p/mm Target

Fig. 13 Images of Target With Faceplate G 
holographic system are shown below in Fig. 14.

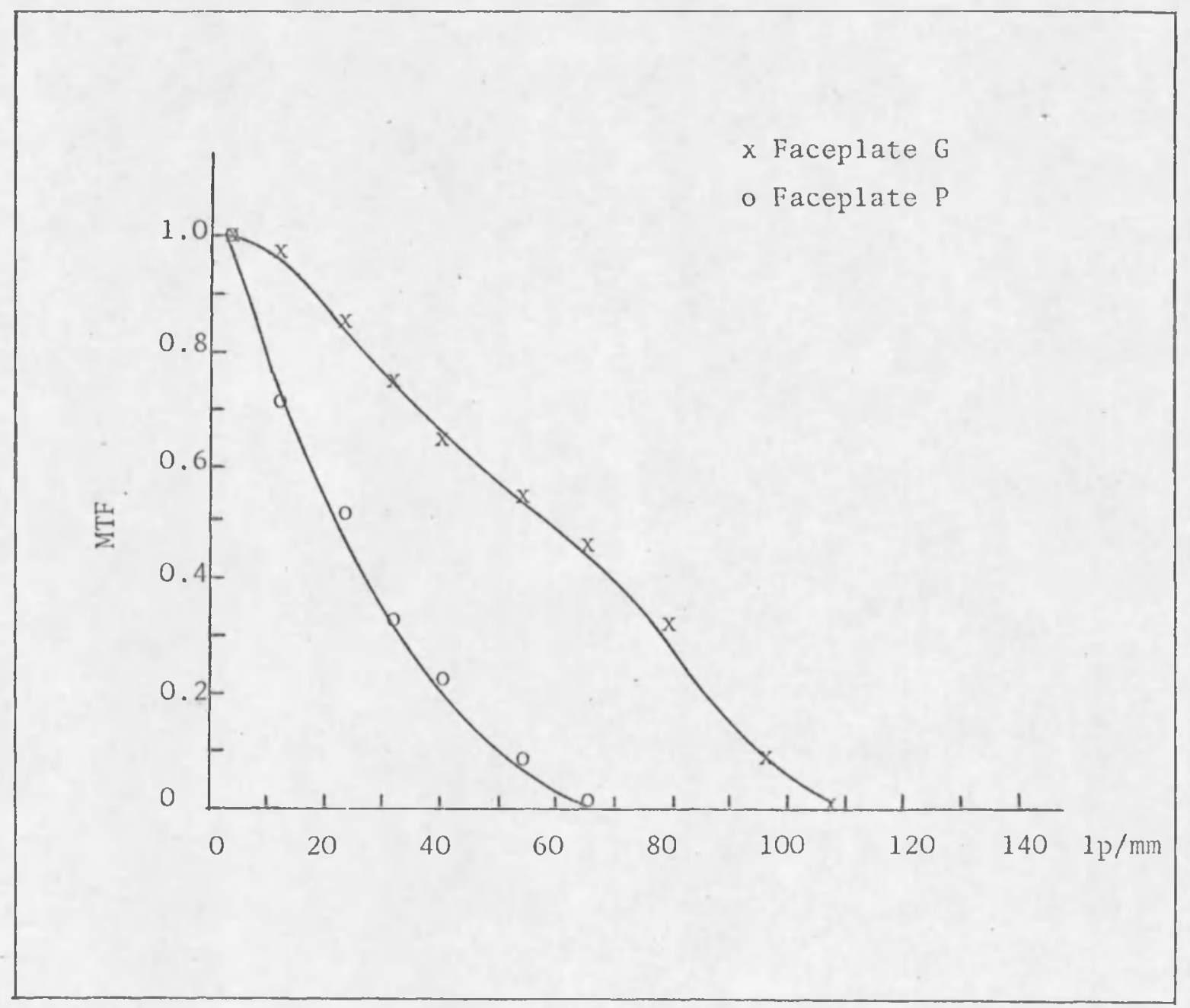

Fig. 14 Statistical MTF

In addition to the statistical MTF, valuable qualitative information can be obtained from the Fraunhofer diffraction patterns. Fig. 15 shows the diffraction pattern from the poor faceplate output hologram. An inspection of the diffraction pattern shown in Fig. 15 reveals the following features. 


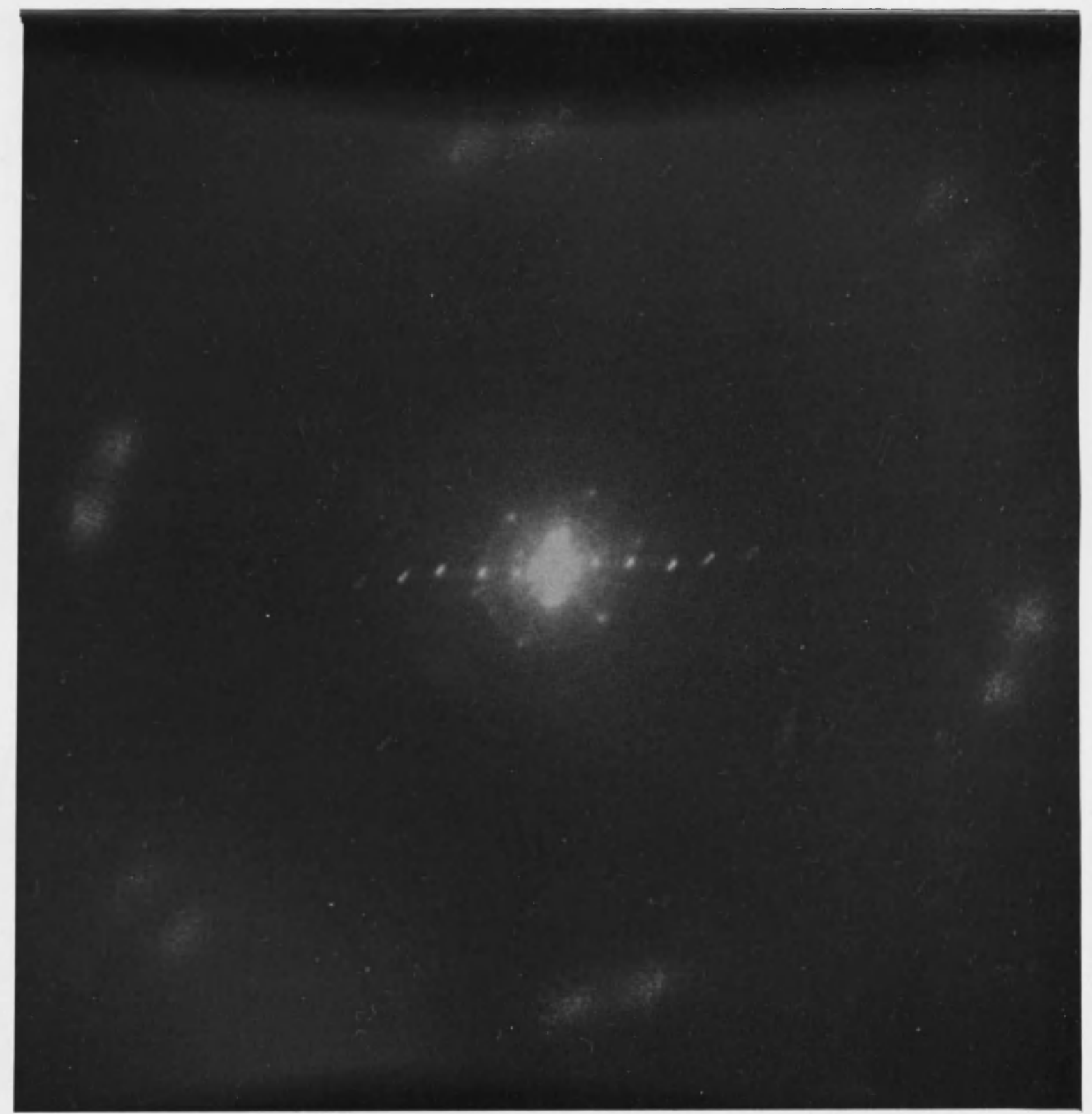

Fig. 15 Fraunhofer Diffraction Pattern of Faceplate P Output Hologram 
At a radius corresponding to a spatial frequency of about 20 Ip/mm, there is a hexagonal diffraction pattern corresponding to the spatial frequency of smaller bundles within the faceplate. The faceplate suffers from poor packing of these smaller bundles.

The six outer dots appearing at the radius corresponding to the spatial frequency of the single fibers are "double dots". There is a rotational distortion of the single fiber arrays.

The six outer dots are not all equidistant from the center, indicating that the individual fibers are not uniformly packed within the bundles.

The sinc ${ }^{2}$ irradiances to be measured go out to a region corresponding to a spatial fxequency of approximately $66 \mathrm{1p} / \mathrm{mm}$ and are rather smeared. The lateral and rotational distortions, poor packing and possibly dead or missing single fibers result in a poor statistical MTF for the faceplate.

\section{Errors of The Experiment}

A comprehensive theoretical error analysis was not performed, but. while components of the system were being designed for optimum pexformance, fifty statistical transfex functions for the same faceplate were generated and analyzed. Relative values of the statistical MTF are repeatable to within $\pm 1 \%$, but a second error factor due to normalization at $1.001 \mathrm{p} / \mathrm{mm}$ is a bit more difficult to analyze since it depends upon whether a good or poor faceplate is being used. A poor faceplate will show a drop of les's than .01 at $1.1 \mathrm{p} / \mathrm{mm}$. Therefore, if a poor faceplate is tested and the statistical MTF normalized to 1.00 
at $1 \mathrm{pp} / \mathrm{mm}$, the absolute exror is within +.06 . The corresponding error for a good faceplate is about +.02 .

\section{Physical Limitations}

The first problem is associated with normalization of the statistical MTF at a spatial frequency which is not zero. With fiber optics it is not correct to assume an approximate statistical MTF value of 1.00 at spatial frequencies of $2-4 \mathrm{lp} / \mathrm{mm}$, so the problem reduces to the construction of a holographic subject to produce an input irradiance of the lowest possible spatial frequency with the least amount of noise in the center of the Fraunhofer diffraction pattern.

Decreasing $S_{1}$, the center-to-center spacing between the reference lens and lens \#1 of the holographic subject, and increasing the focal length of $L_{2}$, the transform lens, Iowers the spatial frequency associated with the first sinc $^{2}$ irradiance in a diffraction pattern (see Fig. 2). The minimum useful value of $S_{I}$ is governed by noise at the center of the measured Fraunhofer diffraction patterns. With $S_{1}$ very sma11, it also becomes much more difficult to build the holographic subject and have the cone of light from the reference lens intersect the cones of light from all of the other eleven lenses. The limitation on increasing the focal length of the transform lens involves an increase in $f /$ number of the system and a reduction of illumination at the hologram plane to such a low level that exposure times for producing the holograms becomes excessively high. Nevertheless, the problems associated with the production of a low spatial frequency sinusoidal irradiance distribution at the fiber optics input plane are manageable and the following technique is effective. 
As much as $50 \%$ of the noise near the first sinc ${ }^{2}$ irradiance of the Fraunhofer diffraction pattern is removed by situating a square aperture at an angle of $45^{\circ}$ with respect to the axis of the sinusoidal patterns recorded on the holograms. This causes the larger secondary irradiances of the sinc distribution centered at the origin of the diffraction pattern to lie along a line $45^{\circ}$ away from the line of narrow sinc ${ }^{2}$ irradiances that are measured. As an example of this technique, Fig. 11 is a diffraction pattern made with a circular aperture, while Fig. 6 and Fig. 8 are diffraction patterns made with a rotated square aperture. 


\section{CHAPTER IV}

\section{COMPARISON OF THE HOLOGRAPHIC METHOD WITH CONVENTIONAL METHODS OF FIBER OPTICS EVALUATION}

Conventional methods used to evaluate fiber optics are classified as either static or dynamic tests. In the static tests an input irradiance distribution is applied at the fiber optics input surface and transferred to the output surface where the image appears. The fiber optics element does not move with respect to the input irradiance distribution. In the dynamic test the fiber optics element is moved rapidly in all directions allowing each fiber to cover uniformly an input plane area which has the dimensions of a few fiber diameters. The image is recorded as a time average, integrated irradiance distribution where the time duration for recording the image is chosen to enable the scanning motion to cover uniformly the small input plane area.

\section{Static Tests}

Some quantitative tests of static fiber optics elements resort to the evaluation of line spread functions and edge responses while less quantitative static tests involve an examination of resolution target: images. These static evaluations include the calculation of transfer functions from edge response and line spread data, but for a single faceplate these transfer functions are found to vary wildly depending upon how the object is located with respect to fiber striations 
(Yanagi and Jeskie 1969). Certainly, wildly varying results are expected since the transfer functions are calculated from response data secured in a small mon-isoplanatic region. A11 of the conventional quantitative tests of fiber optics suffer from the general problem of looking at a smal1 number of single fibers (Kapany 1967, pp. 397-410). The faceplates $G$ and $P$ used in the holographic testing system were subjected to an edge response test at Mosaic Fabrications, Division of Bendix Corporation. From edge response data, a static transfer function was generated for each faceplate and compared to the holographically determined statistical MTF as shown in Fig. 16.

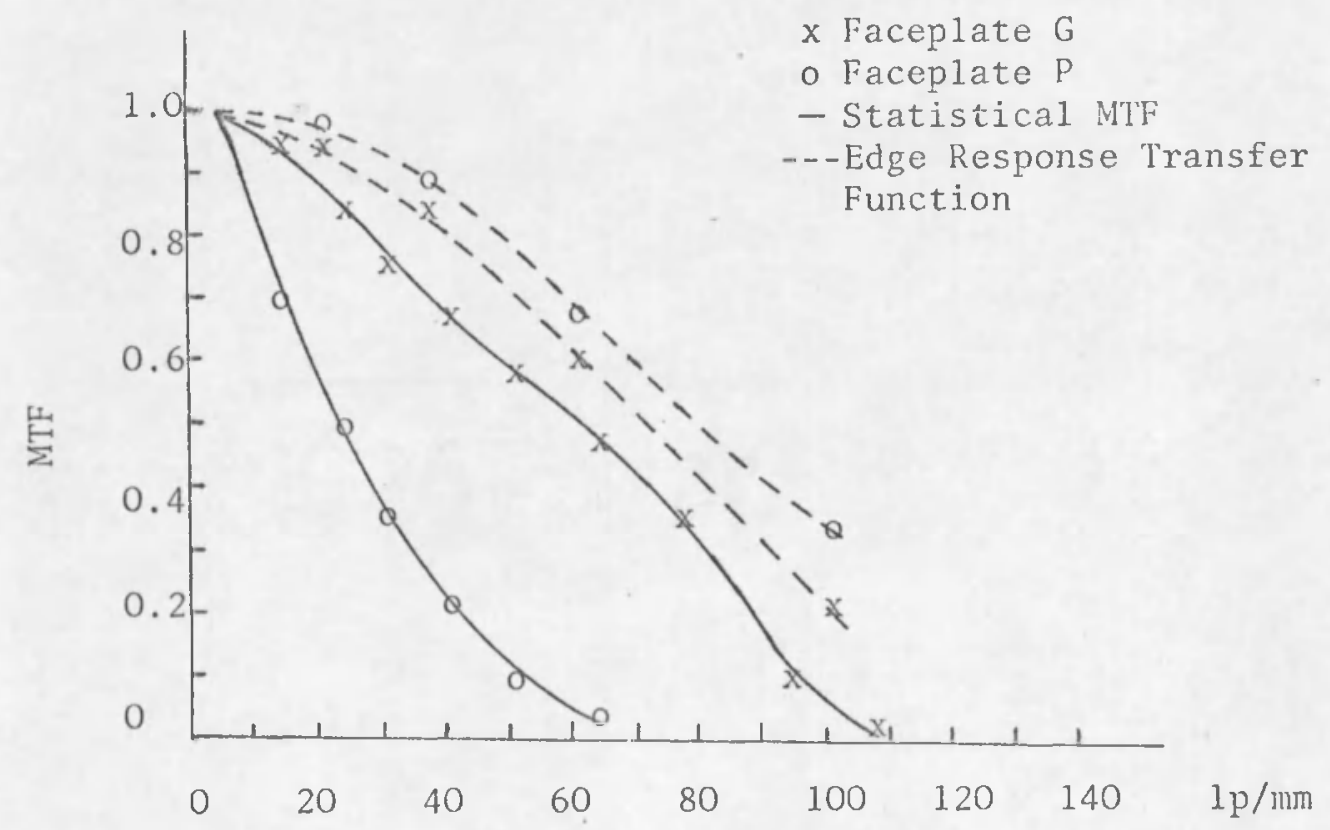

Fig. 16 Edge Response Transfer Function 
The edge response transfer function indicates that both faceplates are of very high quality. In fact, faceplate $P$ shows a higher edge response transfer function than faceplate $G$, while the statistical MTF of faceplate $\mathrm{P}$ clearly shows its poorer performance. The failure of the edge response test is explained by the concept of testing a small non-isoplanatic region of the faceplate.

\section{Dynamic Tésts}

The time-average integrated image obtained with a dynamic test of a fiber optics element does not show the honeycomb structure of the output surface (Kapany 1967, pp. 88-100). A good dynamic scan allows each resolution element to cover uniformly a small input plane area the size of a few resolution elements and thus removes the inherent nonisoplanatic property of the fiber optics surface in this sma11 area. An average spread function can be recorded for this isoplanatic region and the maximum theoretical MTF of a small isoplanatic region created by dynamic scanning is just

$$
\operatorname{MTF}(\varepsilon)=\frac{2 \mathrm{~J}_{1}(\pi \varepsilon \mathrm{d})}{\pi \varepsilon \mathrm{d}},
$$

where d is the fiber diameter (Yanagi and Jeskie 1969; Drougard 1964). The dynamic test and associated dynamic MTF are theoretically acceptable because of the existence of a small isoplanatic region but the dynamic test does not correctly relate the performance of the whole fiber optics element. Large scale distortions in the fiber optics 
such as those caused by poor packing of the smallex bundles or a poor pressing operation do not affect the dynamic MTF.

\section{Statisticà MTF}

The statistical MTF is a measure of the average performance of a fiber optics element over a large portion of its surface. With the evaluation of a stochastic image the non-isoplanatic property of the fiber optics disappears and the statistical MTF is a consistent and meaningful function which can be used to evaluate the performance of a given faceplate. The statistical MTF measured experimentally by holographic techniques is sensitive to factors such as fiber size, fiber spacing, cross talk and distortions between input and output surfaces. If a particular fiber optics element has a good statistical MTF then it is, without question, a good image transferring element. On the other hand, if a faceplate exhibits a poor statistical MTF, an examination of the diffraction pattern from the output hologram can yield information as to what is wrong with the particular el,ement. Since the statistical MTF is repeatable, sensitive to most fiber optics imperfections, and relates the average performance of the entire fiber optics element, it is an attractive method of evaluating the image forming capability of a fiber optics element. 


\section{REFERENCES}

Bracewe11, Ron. The Fourier Transform and Its Applications. New York: McGraw Hi 11 Book Company, 1965, pp. 381.

Drougard, Renee. "Optical Transfer Properties of Fiber Bundles," J.O.S.A., Vol. 54, Number 7, July 1964, pp. 907-914.

Goodman, Joseph W. Introduction to Fourier Optics. San Francisco: McGraw Hi11 Book Company, 1968, pp. 287.

Kapany, N. S. Fiber Optics. New York: Academic Press, Inc., 1967, pp. 429 .

Kozma, Adam. "Photographic Recording of Spatially Modulated Coherent Light," J.O.S.A., Vol. 56, Number 4, April 1966, pp. 428-432.

Potter, Robert J. "Transmission Properties of Optical Fibers," J.G.S.A., Vo1. 51, Number 10, October 1961, pp. 1079-1089.

Smith, Howard M. Principles of Holography. New York: Wiley-Interscience, 1969 , pp. 239.

Yanagi, John, and Jeskie, Francis. "Applications of Fiber Optics," SPIE Journal, Vol. 7, September 1969, pp. 165-167. 